\title{
Liver haemangiomas and consumptive hypothyroidism in association with three cutaneous haemangiomas
}

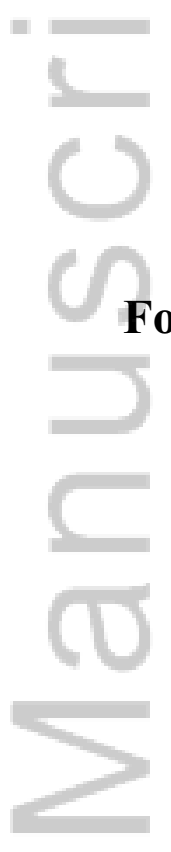

\author{
Instructive Case
}

For submission to Journal of Paediatrics and Child Health

${ }^{1}$ Department of General Medicine, ${ }^{2}$ Deparment of Dermatology, and, ${ }^{3}$ Department of Endocrinology and Diabetes, The Royal Children's Hospital, 50 Flemington Road, Parkville, Victoria, Australia 3052

Correspondence: Dr Trung Nguyen, Department of General Medicine, 3 West Clinical Offices, 50 Flemington Road, Parkville, VIC, Australia, 3052. Phone:+61 39345 5522; fax: +61 39345 4751; email: trung.nguyen@rch.org.au

This is the author manuscript accepted for publication and has undergone full peer review but has not been through the copyediting, typesetting, pagination and proofreading process, which may lead to differences between this version and the Version of Record. Please cite this article as doi: $10.1111 /$ jpc.13671

This article is protected by copyright. All rights reserved. 


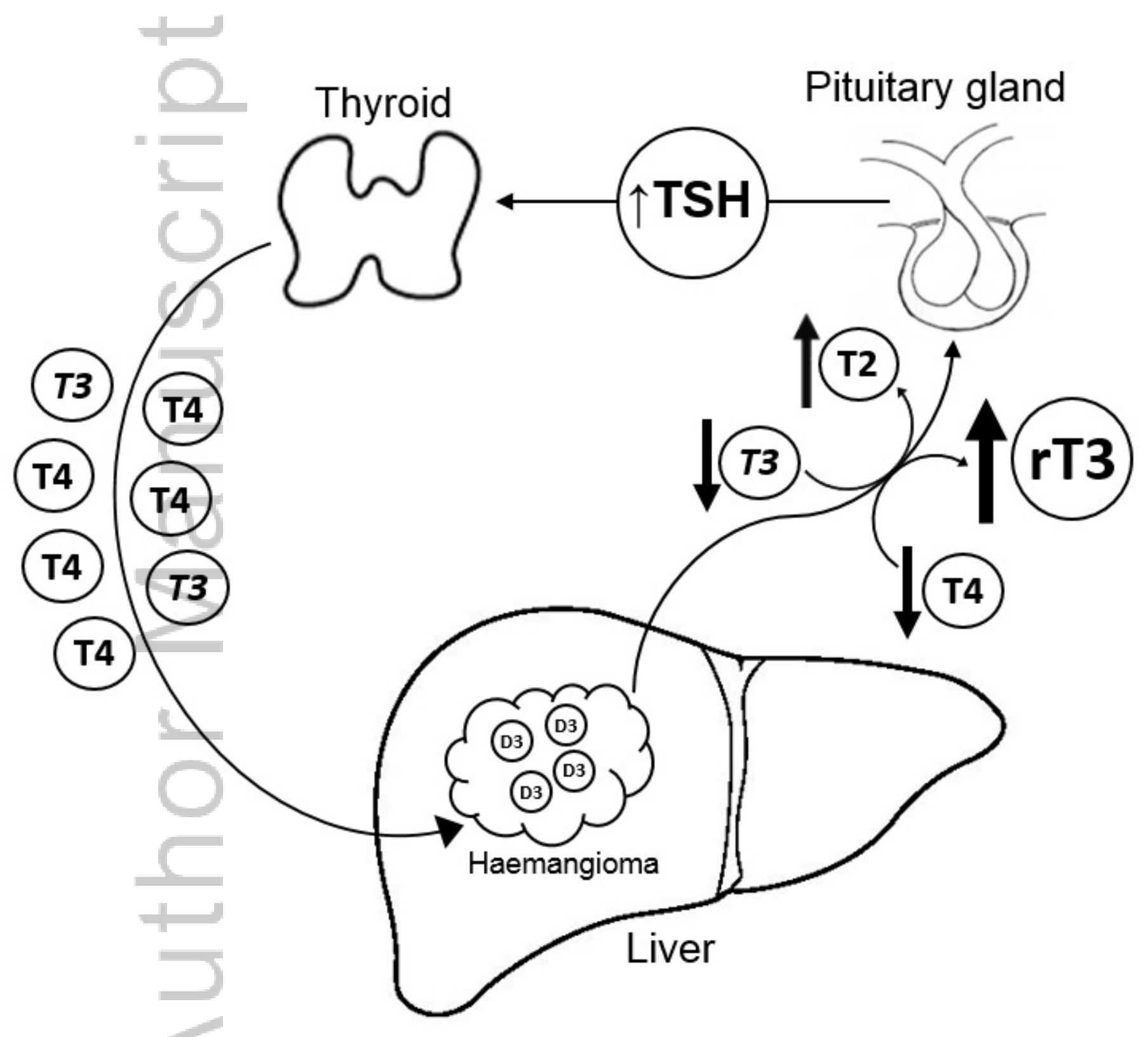

Image - Liver haemangiomas and consumptive hypothyroidism in association with three cutaneous haemangiomas.jpg 


\section{Liver haemangiomas and consumptive hypothyroidism in association with three cutaneous haemangiomas}

\section{Key points}

- The presence of visceral haemangiomas, as well as their associated complications, should be considered in infants with more than one cutaneous haemangioma.

- Consumptive hypothyroidism is a rare complication of hepatic haemangiomas, and, if thyroid hormone replacement is required, high doses are often needed due to the consumptive nature of the hypothyroidism.

- Hypothyroidism usually resolves with involution or treatment of the hepatic haemangiomas.

Infantile haemangioma is the most common vascular tumour of infancy, occurring in approximately 5 percent of infants. ${ }^{1}$ The natural progression of cutaneous haemangiomas is well described. Often absent at birth, they demonstrate an early proliferative phase characterised by appearance and rapid growth during the first three months of life. A slower, more gradual proliferation can continue at times up until six to nine months of age. Involution and regression occur over a variable time frame, commonly ending at approximately three to five years of age. ${ }^{1}$ Single cutaneous haemangiomas are more common than multiple cutaneous haemangiomas. ${ }^{1}$ Given the high likelihood of spontaneous involution, most cutaneous haemangiomas do not require intervention. The minority requiring treatment are those that demonstrate ulceration, result in disfigurement, or risk functional impairment (for example, around the airways). ${ }^{1,2}$

Visceral haemangiomas can also occur and the liver is the most commonly affected extracutaneous site. Complications of hepatic haemangiomas include hepatomegaly, high-output cardiac failure due to shunting, obstructive jaundice ${ }^{1}$, and hypothyroidism. ${ }^{3}$ The presence of multiple cutaneous haemangiomas is widely regarded as a marker of hepatic haemangiomas, and most recommendations suggest screening for hepatic haemangiomas with abdominal ultrasound in infants with five or more cutaneous haemangiomas. ${ }^{1,2,3,4}$ These recommendations are supported by a study that screened infants under six months of age with one or more cutaneous haemangiomas with ultrasound, and found that no infant with fewer than five cutaneous haemangiomas had hepatic involvement. ${ }^{5}$

We describe the case of an infant with only three cutaneous haemangiomas who was found to have multiple liver haemangiomas associated with the rare complication of consumptive hypothyroidism. This case acts as a useful clinical vignette with which to highlight the importance of considering rare complications in infants with more than one cutaneous haemangioma. 


\section{Case Report}

A two-month-old boy with three cutaneous haemangiomas was referred to the Emergency Department for an incidental finding of massive hepatomegaly. He had been born at term gestation, and antenatal and birth history were unremarkable. Newborn screening test was normal. At one week of age, he developed three cutaneous haemangiomas (right forehead, peri-umbilical region and plantar aspect of his right foot). All three haemangiomas had been growing in size with no ulceration. He was otherwise well with good growth $\left(6.44 \mathrm{~kg}, 80^{\text {th }}\right.$ percentile at two months of age $)$. There were no concerns with development.

On presentation he appeared well with normal vital signs. Heart sounds were dual with an ejection systolic murmur grade 3/6 loudest in the left upper sternal edge with no radiation and no displacement of the cardiac impulse. Lung auscultation was normal. Liver edge was palpable $5 \mathrm{~cm}$ below the costal margin. No hepatic bruit was appreciable. There was no splenomegaly and abdomen was soft. He was not jaundiced and there was no bruising. Femoral pulses were present and normal.

Liver function testing demonstrated only mild elevation of gamma-glutamyl transferase level (336 IU/L, normal $<225$ IU/L), with normal alanine aminotransferase and alkaline phosphatase levels. Full blood examination, electrolytes, coagulation studies and venous blood gas were normal.

Abdominal ultrasound demonstrated multiple, variably-sized, vascular nodules consistent with liver haemangiomas. Given the possible association of hypothyroidism in liver haemangiomas, thyroid function testing was performed which demonstrated an elevated thyroid stimulating hormone (TSH) level of $54 \mathrm{mIU} / \mathrm{L}$ (normal 0.5-4.5 mIU/L), thyroxine (T4) level of $28.6 \mathrm{pmol} / \mathrm{L}$ (normal 10-25 $\mathrm{pmol} / \mathrm{L}$ ), and triiodothyronine (T3) level of $6.2 \mathrm{pmol} / \mathrm{L}$ (normal 4.2-8.3pmol/L). Echocardiogram showed mild aortic stenosis only.

Given the possibility of spontaneous regression of the haemangiomas, the decision was made not to commence treatment for the haemangiomas. In addition, given T4 level was not low despite an elevated TSH, no treatment for hypothyroidism was commenced. He remained well and was discharged home.

Review at three months of age revealed two new cutaneous haemangiomas (one on the chest and one on his abdomen). The initial haemangiomas had continued to enlarge with no ulceration. There was some improvement in TSH to $39.5 \mathrm{IU} / \mathrm{L}$. T4 was $23.8 \mathrm{pmol} / \mathrm{L}$ and T3 was $7.6 \mathrm{pmol} / \mathrm{L}$. At this stage, he was commenced on oral propranolol due to increasing number and size of haemangiomas along with the complication of liver haemangiomas and hypothyroidism. Repeat echocardiogram was unchanged. 
At seven months of age, the cutaneous haemangiomas remained the same size despite propranolol. Thyroid function testing, however, demonstrated normal TSH of 3.7 IU/L with normal T3 and T4 levels. He remained well with normal growth and development.

\section{Discussion}

The unique features of this case were the presence of multiple hepatic haemangiomas with only three cutaneous haemangiomas, and the rare complication of consumptive hypothyroidism.

Consumptive hypothyroidism is a rare paraneoplastic syndrome that can occur in infants with hepatic haemangiomas. It results from the abnormal over-expression of type 3 iodothyronine deiodinase (D3), a selenoenzyme responsible for inactivation of thyroid hormone molecules by converting T4 to reverse T3 (rT3), and T3 to 3,3'-diiodothyronine (T2). ${ }^{6,7,8}$ Under normal conditions, D3 regulates circulating levels of thyroid hormone and is restricted to the central nervous system, skin, and some endocrine glands. ${ }^{7}$ However, biopsies have demonstrated over-expression of D3 by hepatic haemangioma tissue. ${ }^{6,7}$

Consumptive hypothyroidism can occur when the catabolic activity of D3 exceeds the thyroid gland's ability to synthesise thyroid hormone. ${ }^{6,7}$ This is demonstrated in Figure 1. D3 activity appears to increase most rapidly during the proliferative phase of haemangiomas, and risk of thyroid hormone degradation is most severe during this time. ${ }^{7}$ Our patient presented with subclinical hypothyroidism manifesting with an elevated TSH and normal T3 and T4, but he had no signs of hypothyroidism. His TSH was elevated in response to degradation of thyroid hormone by D3, but thyroid hormone levels remained adequate as his thyroid was functionally normal. As a result, no treatment was initially commenced. However, the importance of monitoring thyroid function and regular clinical assessment is paramount given the risk of the consumption of thyroid hormone worsening with loss of sufficient T3 and T4, and the consequence of adverse neurocognitive development of untreated hypothyroidism in infancy. Unfortunately, rT3 level had not been sent to the laboratory when our patient initially presented to hospital. However, the rT3 level would be expected to be elevated in consumptive hypothyroidism due to increased conversion of T4 to rT3. An elevated rT3 level is useful for differentiating consumptive hypothyroidism from other causes of hypothyroidism.

Other important differences exist between consumptive hypothyroidism and conventional congenital hypothyroidism. Consumptive hypothyroidism is not usually detected by newborn screening as haemangiomas are typically not apparent at birth. ${ }^{8}$ Treatment of consumptive hypothyroidism can be difficult as exogenous hormones are inactivated by D3, and high doses of thyroid hormone replacement are typically needed when compared to conventional hypothyroidism. ${ }^{6}$ The use of 
intravenous thyroxine has been described in particularly severe cases of consumptive hypothyroidism. ${ }^{6,7}$

Consumptive hypothyroidism appears to resolve with resolution of hepatic haemangiomas. ${ }^{7,8}$ Although spontaneous involution of haemangiomas can occur, treatment of hepatic haemangiomas is sometimes required. Treatment modalities that have been described for hepatic haemangiomas complicated by consumptive hypothyroidism include pharmacological modalities (for example, oral propranolol, which is considered to be first-line therapy for cutaneous haemangiomas, and corticosteroids), and embolisation, ligation of the hepatic artery, resection and liver transplantation for severe cases in which there is inadequate response of the hepatic haemangiomas to non-surgical management. ${ }^{\text {? }}$

This case highlights the importance of considering the presence of hepatic haemangiomas even in patients who have fewer than five cutaneous haemangiomas. Screening for liver involvement in infants with any number of cutaneous haemangiomas should be considered if hepatomegaly is present. Moreover, the possibility of consumptive hypothyroidism should be considered in infants with liver haemangiomas, and ongoing monitoring of the progress of these infants based on clinical and biochemical grounds is vital given the adverse effects of untreated hypothyroidism.

\section{Multiple Choice Questions}

1. Which of the following histochemical markers is associated with infantile haemangiomas?
a. Anti-thyroid transcription factor-1 (TTF-1)
b. Epithelial membrane antigen (EMA)
c. Glucose transporter 1 (GLUT-1)
d. Paired box gene 8 (PAX8)
e. Angiogenin (ANG)

Answer: c. Infantile haemangiomas involve proliferation of endothelial-like cells containing the GLUT-1 marker. Both infantile haemangiomas and placental tissue contain GLUT-1 markers. Haemangiomas appear to originate from the placenta, and hypoxic insults to the placenta appear to result in vascular proliferation of infantile haemangiomas. Infants with history of low-birth weight and advanced maternal age are more likely to have haemangiomas, suggesting the role of hypoxia during transition to postnatal life in development of haemangiomas.

2. Which of the following is NOT a recognised potential side effect of propranolol?
a. Bradycardia
b. Hypoglycaemia
c. Sleep disturbance 

d. Hypertension
e. Wheeze

Answer: $d$. As a healthcare provider, awareness of potential adverse effects of prescribed medications is essential. Careful note of baseline heart rate, blood pressure, blood glucose levels, sleep patterns and presence of pre-existing bronchospasm is essential prior to prescribing propranolol to infants, and will enable accurate dose adjustment and ensure avoidance of side effects. Propranolol is well tolerated in term infants, but should be administered with caution in pre-term infants as there is a higher incidence of bradycardia and hypotension.

3. Which of the following investigation findings would be most typical of consumptive hypothyroidism?
a. Elevated levels of serum thyroglobulin antibody
b. Increased TSH and rT3 levels
c. Reduced TSH, T3 and T4 levels
d. Elevated thyroid uptake on a nuclear medicine scan
e. Presence of a sublingual thyroid on an ultrasound scan

Answer: b. The presence of elevated levels of TSH and rT3 associated with rapid proliferation of haemangiomas is most suggestive of consumptive hypothyroidism. Thyroid antibodies are not responsible for the mechanism of hypothyroidism associated with haemangiomas, reduced TSH level is suggestive of a central (hypothalamic or pituitary) cause of hypothyroidism, abnormal thyroid uptake on a nuclear medicine scan is suggestive of dyshormonogenesis as a cause of hypothyroidism, and a sublingual thyroid is suggestive of an ectopic thyroid gland.

\section{References}

1. Laken PA. Infantile hemangiomas: pathogenesis and review of propranolol use. Adv. Neonatal Care 2016; 16: 135-142.

2. Leaute-Labreze C, Prey S, Ezzedine K. Infantile haemangioma: part II. Risks, complications and treatment. J. Eur. Acad. Dermatol. Venereol. 2011; 25: 1254-1260.

3. Haggstrom AN, Drolet BA, Baselga E, et al. Prospective study of infantile haemangiomas: clinical characteristics predicting complications and treatment. Pediatrics 2006; 118: 882-887.

4. Frieden IJ, Haggstrom AN, Drolet BA, et al. Infantile hemangiomas: current knowledge, future directions. Proceedings of a research workshop on infantile hemangiomas. Paediatr. Dermatol. 2005; 22: 383-406. 
5. Horii KA, Drolet BA, Frieden IJ, et al. Prospective study of the frequency of hepatic hemangiomas in infants with multiple cutaneous infantile hemangiomas. Paediatr. Dermatol. 2011; 28: 245-253.

6. Jassam N, Visser TJ, Brisco T, Bathia D, McClean P, Barth JH. Consumptive hypothyroidism: a case report and review of the literature. Ann. Clin. Biochem. 2011; 48: 186-189.

7. Luongo C, Trivisano L, Alfano F, Salvatore D. Type 3 deiodinase and consumptive hypothyroidism: a common mechanism for a rare disease. Front. Endocrinol. 2013; 4: 115.

8. Cho YH, Taplin C, Mansour A, et al. Case report: consumptive hypothyroidism consequent to multiple infantile hepatic haemangiomas. Curr. Opin. Pediatr. 2008; 20: 213-215.

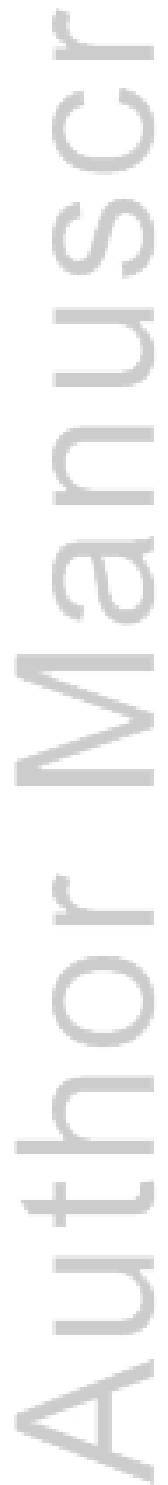




\section{University Library}

\section{- M M N E R VA A gateway to Melbourne's research publications}

Minerva Access is the Institutional Repository of The University of Melbourne

Author/s:

Trung, N;Orchard, D;Zacharin, M

Title:

Liver haemangiomas and consumptive hypothyroidism in association with three cutaneous haemangiomas

Date:

2017-12-01

Citation:

Trung, N., Orchard, D. \& Zacharin, M. (2017). Liver haemangiomas and consumptive hypothyroidism in association with three cutaneous haemangiomas. JOURNAL OF PAEDIATRICS AND CHILD HEALTH, 53 (12), pp.1226-1228. https://doi.org/10.1111/ jpc. 13671.

Persistent Link:

http://hdl.handle.net/11343/293232 